\title{
Reduction of wind turbine noise annoyance: an operational approach
}

\author{
Annelies Bockstael, Luc Dekoninck, Arnaud Can, \\ Damiano Oldoni, Bert De Coensel, Dick Botteldooren
}

February 3, 2012

\begin{abstract}
This paper investigates the relationship between wind turbine noise annoyance, exposure indicators, operational characteristics and environmental variables. A six-month field experiment at an industrial site near a residential area includes regular on-line annoyance reports, continuous $1 / 3$-octave band noise level registrations, periodic sound recordings, data on electricity production per minute and meteorological observations. Here the risk of high annoyance does not only depend on the angular blade velocity, but also on the wind turbines' nacelle position relative to the location of the dwellings, i.e. the wind direction. This directivity effect can be captured when noise parameters such as the background noise level caused by other sources and a so-called fluctuation-indicator are introduced, the latter calculated from the 1/3-octave band spectra to quantify the periodic part of wind turbine noise. In addition, the calculated turbine's specific emission levels are closely related to the angular blade velocity, and an important parameter to predict the risk of high annoyance. Finally, these results suggest that operational restrictions based on wind direction together with the angular blade velocity might help to reduce noise annoyance while preserving cost-effectiveness.
\end{abstract}

\section{Introduction}

Growing ecological awareness has increased energy production by wind turbines [1, 2], but the installations themselves affect the surrounding landscape and soundscape. In this regard, noise is most prominent in the closer vicinity of the turbines [2], making the issue especially important in densely populated areas. Moreover, wind turbine noise annoyance occurs to a higher degree than other sources of community noise at the same average noise exposure level [3-7] and complete energetic masking is difficult to obtain [6, 8].

Annoyance assessment and management requires a valid exposure-effect relationship for which a classical exposure parameter is the A-weighted equivalent sound pressure level ( $\left.L_{\mathrm{Aeq}}\right)$ [3] or $L_{\mathrm{den}}$ [7], but more exposure indicators might be needed. Laboratory listening tests for qualitatively different wind turbine noise fragments with equalized $L_{\text {Aeq }}$ give different annoyance ratings [9] and qualitative descriptions 'swishing', 'whistling', 'resounding' and 'pulsating/throbbing' appear to a very good extent correlated with annoyance by wind turbine noise [10]. No relationship is found between varying annoyance response and psychoacoustic parameters like sharpness, loudness, roughness, fluctuation strength or modulation [9]. 
To manage noise annoyance, carefully designed control strategies are needed, especially when wind turbines are introduced in highly populated regions. Technical modifications have been studied widely for this. In addition, in critical situations, operational restrictions may reduce noise annoyance, but then cost-effectiveness should be preserved as much as possible. Here, good policy needs thorough insight in relevant noise characteristics and easily measurable parameters to steer the turbines in practice.

This article investigates the relationship between wind turbine noise annoyance and exposure indicators, operational characteristics and context variables. Alternative noise immission indicators other than $L_{\mathrm{Aeq}}$ and the classical psychoacoustic parameters will be linked to annoyance and to operational and meteorological data. If a significant relationship can be found between on the one hand the alternative noise immision parameters and on the other operational and/or meteorological data, it proves the very tight relationship between the noise immission measurements and the (actual) wind turbine noise emission. Moreover, operational and meteorological information can then be used to steer the wind turbine rather than noise immission measurements that are more difficult to organize.

The strategy described above is applied in a field experiment carried out at a specific wind turbine site in the Flemish part of Belgium with a long history of noise complaints due to wind turbine noise. Long-term and detailed noise measurements have been collected and analyzed together with operational and meteorological information. Annoyance records have been gathered via an on-line web application filled in by volunteers living in the neighborhood. Hence, the current approach distinguishes itself from epidemiological studies by more detailed data gathering and from a laboratory setup by higher ecological and contextual validity.

To sum up, the three major research questions are (1) to link wind turbine noise annoyance to meteorological and operational parameters, enabling the selection of practical usable steering parameters, (2) to investigate for noise exposure the influence on annoyance as well as the relationship with the meteorological and operational parameters mentioned above, helping to understand better the mechanisms behind wind turbine noise annoyance and (3) to apply these research data on a case study, evaluating theoretical predictions in a real-life setting.

In the manuscript, the most relevant theoretical insights in wind turbine noise will be sketched first, followed by the test setup, results and points for discussion.

\section{Origins of wind turbine noise}

In general, wind turbines generate both mechanical and aerodynamic sound, but current technology has made the mechanical noise less important by reducing it below the level of the aerodynamic noise in normal operational conditions [11]. This is especially true for the direct shaft machine studied here. Aerodynamic noise is caused by the flow of air around the wind turbine blades [12]. In high power wind turbines-which are of concern in this paper-turbine blade form has been optimized for efficiency and low noise level. From the vast amount of research on this topic, the aspects of interest in this field experiment are summarized.

Aerodynamic noise strongly depends on flow speed and thus on rotation speed of the wind turbine. Trailing edge noise for example is theoretically proportional to the fifth power of flow velocity. Since wind turbine blades are typically pitched depending on the wind velocity, the dependence on rotation speed might deviate, but still a strong increase of noise level with rotation speed is expected. In earlier and smaller wind 
turbines, trailing edge bluntness sometimes led to periodic vortex shedding and resulted in tonal noise. However, tonality is less likely to be observed for the modern wind turbine under study.

The turbulent flow around the wind turbine blade and this flow's interaction with the blade leads to a very directive noise emission from the tip area of each blade [13]. This could result in noise emission directivity by the wind turbine as a whole and thus a dependence of the immission on wind turbine orientation and wind direction. In addition, blade noise emission directivity could result in periodic fluctuation of noise immission at ground level. Directivity and fluctuation strength combine to a non-trivial directivity dependence of immission at ground level.

Oerlemans [13, 14] presents a theoretical model that explains directivity observed in the field. In summary, noise levels are expected to be low in the plane of the wind turbine blades, but fluctuation strength is strong, leading to an expected maximum in fluctuation strength at angles close to but not coinciding with the plane of the blades. According to Oerlemans [13] these fluctuations are approximately 2 to $3 \mathrm{~dB}$, but can be quite easily detected by the human listener at levels of 1 to $2 \mathrm{~dB}$ below the background noise [11]. In addition, the effect might increase when different turbines are rotating together [15]. In the direction of the turbine axes, the noise level caused by the boundary flow on the blades is high but does not fluctuate with the position of the blades. Additionally, the directivity of wind turbine noise will be further modified by propagation effects such as ground, obstacles, and so on, since the source height at maximum level is different in different directions.

In addition to the noise caused by the mere presence of the wind turbine blade in an otherwise perfect flow, turbulence in the atmospheric boundary layer hitting the wind turbine may cause additional noise. This contribution to the overall noise level also has a broad spectrum but a lower frequency content than the blade induced noise and is mainly emitted along the axis of the wind turbine [13]. A complex dependence on meteorological conditions and physical surroundings of the wind turbine noise can be expected. Malfunction of mechanical components and small defects on the wind turbine blades may cause additional noise, but it was confirmed that this is not the case in the current setup.

Although Van den Berg [16] has shown that actual (night-time) atmospheric conditions influence the measured sound levels, only very little effect of wind and temperature gradients on propagation is expected at distances up to several hundred meters from $100 \mathrm{~m}$ tall high power wind turbines [17]. Due to the height of the source, the ground effect is also limited and atmospheric absorption has an observable but rather small effect [18].

\section{Materials and method}

\subsection{Description of test site and wind turbines}

This study's test site is localized in a quite urbanized area in the Flemish part of Belgium. The landscape is mainly flat with one two-by-two-lane road and several smaller roads, a factory site and a residential area with all free-standing houses. Three wind turbines have been erected on the factory site's vacant ground between the industrial buildings and the housing, the closest at about $270 \mathrm{~m}$ from the first dwellings. A schematic overview is drawn in Figure 1

The wind turbines have a rotor diameter of $82 \mathrm{~m}$ and a hub height of $90 \mathrm{~m}$ above the 


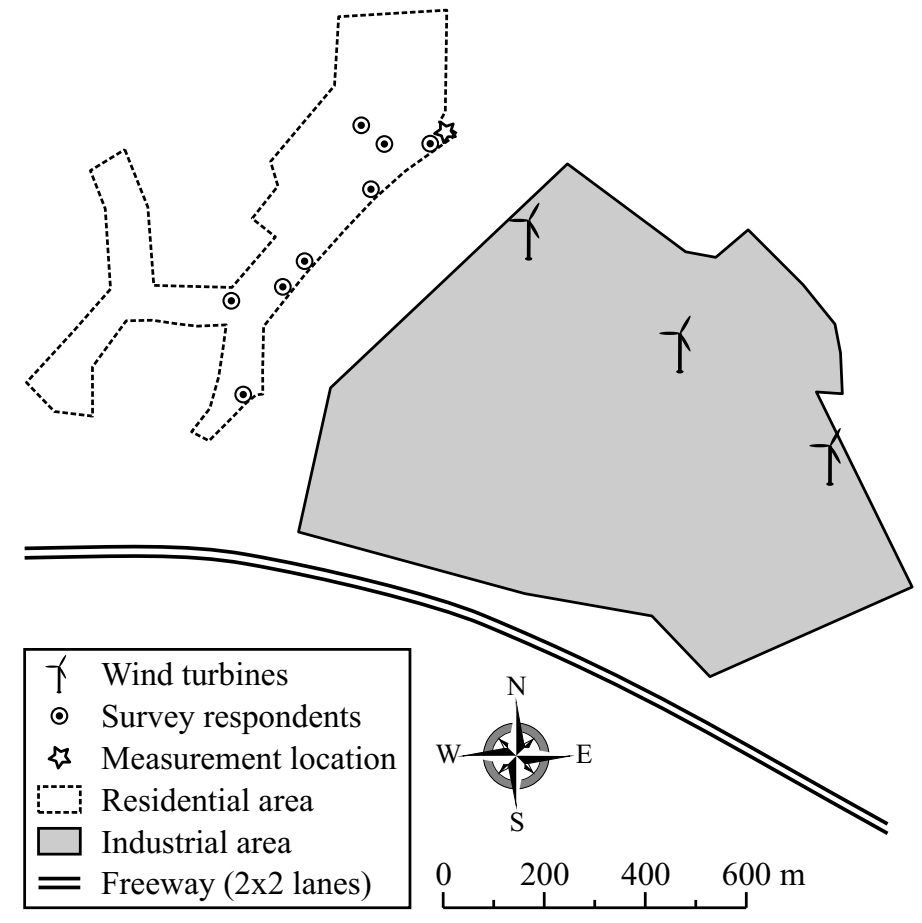

Figure 1: Schematic overview of the test site with the residential area and the major sources of background noise: the two-by-two lane road, the factory and the three windturbines 
ground. The upwind rotor with active pitch control has 3 blades and rotates at 6 to 19.5 rotations per minute, the rated power is $2 \mathrm{MW}$. For these direct drive wind turbines with independently controlled blade pitch, mechanical noise due to rotating parts is unlikely to be observed since there is no potentially noisy gear box.

Following previous complaints from the neighbors, two operational regimes are mostly used: unrestricted operation during the day (7h-19h) and restricted to $600 \mathrm{~kW}$ (or approximately 12 rotations per minute) at night (19h-7h). In addition, the closest turbine is stopped when cast shadows of moving blades could cause flickering light inside the houses.

\subsection{Noise measurements}

\subsubsection{Measurement setup}

The aim of the measurements is to find noise indicators that capture as closely as possible the perceived noise annoyance. Measurements have been performed in the backyard of one of the houses closest to the turbines (i.e. at about $270 \mathrm{~m}$ from the closest installation), meaning that there are no buildings between the closest turbine and the two different measurement points. The first microphone (from the 24th of February until the 26th of April) has been set at $4 \mathrm{~m}$ height at $1 \mathrm{~m}$ of the corner of a garage. The measurement point is close to some trees and other evergreen plants, hence a lot of wind induced vegetation noise is present at relative low wind speeds at ground level.

The second point (from the 7th of April until the 15th of August) has been chosen near the north side of the house close to the bedroom window of the residents, at $1.40 \mathrm{~m}$ height and $1 \mathrm{~m}$ from the façade. The microphone is shielded from the wind and some of the background noise by the house and a wooden frame closing the backside of the garden, at this location minimal masking can be expected.

\subsubsection{Measurement equipment}

The measurement equipment is a Sinus Messtechnic Swing 4 channels measurement system using the SAMURAI 1.7 software. The equipment is setup to measure continues $\frac{1}{3}$-octave bands at subsecond timesteps ( $\frac{1}{8}$ seconds) and to record sound for 1 minute every 15 minutes. To achieve the recording, a trigger signal was put on an output channel, which is measured on a second input channel. The setup is calibrated with a Svantek calibrator $(1 \mathrm{kHz}, 94 \mathrm{~dB})$.

\subsection{Operational and meteorological parameters}

The operational parameters of the wind turbine closest the housing have been made available by the wind turbine company, in particular the angular blade velocity and the electricity production. In addition, the wind speed at hub height and the nacelle position-strongly correlated to the wind direction-have been provided. Meteorological data like temperature and relative humidity have been retrieved from a permanent weather station a few kilometers from the actual test site.

\subsection{Annoyance assessment}

An on-line web-application (in Dutch) has been set up such that the neighbors could report their annoyance from the 10th of March until the 20th of June by simply answering the question 'How severely are you annoyed by the noise of the wind turbines at 
this moment?' with 'not at all', 'slightly', 'moderately', 'highly' or 'extremely'. This question is a slightly adapted version of the ISO standard annoyance question with standardized five-point answering scale [19]. The web-application is preferred over retrospective questionnaires to enable a direct link between instantaneous daytime annoyance, exposure, operational and meteorological data.

Through a door-to-door campaign in the area, eight families willing to participate have been found. They all have been explained personally how to use the webapplication. The reporting period ended by a debriefing of the regular reporters to gain insight into their reporting strategy and general noise sensitivity, the latter by a Dutch adaptation of the Weinstein's noise-sensitivity scale [20] used previously in large-scale Flemish quality of life studies. The non-respondents on the other hand have been contacted for a short telephonic interview based on the questionnaire described in [21] completed with the Weinstein's scale.

\subsection{Statistical analysis}

All statistical analyses are carried out with the statistical software R. In general, analysis of variance (ANOVA), linear and logistic regression are applied. Before the ANOVA or linear regression outcomes are interpreted, the aptness of the analysis' assumptions are verified by inspecting the standardized and studentized residuals [22]. In case of linear regression, outliers are inspected and influential observations are detected via the Mahalanobis distance, dfbetas, Cook's distance and leverage [22].

For logistic regression assessing the risk of high annoyance, the respondents are included as random factors in mixed-effects logistic models [23, 24], taking into account that one respondent could enter multiple annoyance reports. The regression coefficients of the individual respondents are not shown in the final equations because their particular effect is of very little interest. Outliers are assessed by investigating the standardized residuals and influential observations are detected by computing dfbetas for model parameters, dffits, covariance ratios, Cook's distances and hatvalues, the models' fit is evaluated through ROC curves [22]. Conclusions on variables' contribution to the model are based on the statistical significance of their coefficients and changes in model deviance and AIC (Akaike information criterion)-measures of a model's goodness-offit-when this variable is added [22].

\section{Results}

\subsection{Noise indicators}

\subsubsection{Calculation of percentile noise levels}

When calculating percentile noise levels directly based on $\frac{1}{3}$-octave spectra and $\frac{1}{8}$ second time averaged $L_{\text {Aeq }}$, the statistical evaluation might remove some of the short term fluctuations in the wind turbine noise caused by the blade passing. Therefore the $\frac{1}{8} \mathrm{~s}$ $\frac{1}{3}$-octave band spectra are aggregated to a 5 seconds $L_{\text {Aeq }}$ before percentile levels are calculated. The highest expected frequency of the amplitude fluctuation is $1 \mathrm{~Hz}$ (at 20 rotations per minute given the three blades), hence $5 \mathrm{~s}$ aggregation should conserve the total contribution of the wind turbine. Because production, meteorological condition and background noise can fluctuate quickly over time, a time frame for evaluation of 10 minutes is chosen. This is shorter than the common one-hour evaluation to not lose 
the effect of short but higher emission levels of the turbines. Finally, 10 minute samples with the $1 \%$ percentile level $L_{\mathrm{A} 01}$ exceeding $65 \mathrm{~dB}(\mathrm{~A})$ are excluded since they are strongly influenced by events not related to the wind turbine.

\subsubsection{Additional emission caused by wind turbines}

Extracting the contribution of the wind turbine from the overall noise level is a tedious task since operation of the wind turbine is strongly correlated with wind speed (at hub height) and so is background noise. The contribution of the wind turbine to overall noise levels is extracted from differences in measured levels at the second microphone when the regime of the wind turbine changes: (1) during a forced stand still due to for example avoidance of shadow forming; (2) when the production limitation is applied (19h00) or released (7h00). On the 10-minutes aggregated noise levels and production data gathered over test period, several inclusion criteria are applied to avoid unstable noise levels caused by the actual acceleration/deceleration or changes in wind speed; this results in (1) 107 useful observations during forced stand still and (2) 105 observations for production limits.

For the forced stand still (1), Figure 2 shows noise levels attributable solely to the closest wind turbine as a function of wind turbine angular blade velocity. Here, data are only available up-to 17 rotations per minute because it is very rare that the wind turbine would accelerate from 0 (forced stand still) to 18 rotations per minute within a limited time period (20 minutes). Similar results are found for the production limits (2). For lower wind turbine speed, the sound pressure levels thus obtained are slightly lower than theoretical predictions based on constructor sound power data and theoretical propagation models which for instance amount to $40.3 \mathrm{~dB}(\mathrm{~A})$ at 14 rotations per minute. For higher velocity, the calculated sound pressure levels are slightly higher than the theoretical prediction, $42.8 \mathrm{~dB}(\mathrm{~A})$ at 17 rotations per minute. The calculated levels as function of speed are probably somewhat steeper than the theoretical predictions because of the followed measurement and calculation procedure, also in general theoretical predictions tend to be rather smooth.

The linear regression model with the turbine's specific emission level as a function of angular blade velocity confirms with an adjusted $R^{2}$ of 0.77 the very close relationship between observed noise levels and turbines' operational characteristics.

\subsubsection{Background noise level}

Although wind turbine noise is difficult to be fully energetically masked, background noise like wind induced vegetation sounds or road traffic noise might at least informationally mask the sound from the turbines, hence potentially decreasing perception and annoyance [8].

Background levels are obtained from the first measurement point further from the house: percentile levels $L_{\mathrm{A} 95}$ are selected for observations where the angular blade velocity is lower than 10 rotations per minute-to avoid contribution of the wind turbineand $L_{\mathrm{A} 95}$ is lower than $60 \mathrm{~dB}(\mathrm{~A})$-to omit accidental high values. Average levels are summarized per wind direction in Table 1 .

On this particular site, the major background sources are expected to be vegetation together with the two-by-two-lane road and the factory. Four-way linear regression reveals that the background levels are significantly $(\alpha=0.05)$ related to wind speed, day of week, time of day-due to the diurnal character of traffic and industry-and wind direction-due to the specific organization of the site under study. Since this statistical 


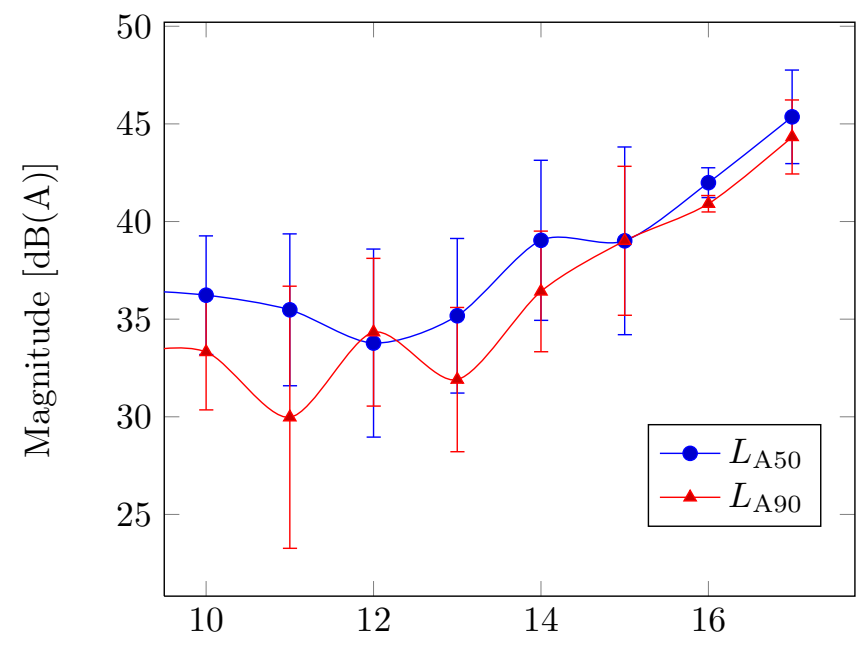

Angular blade velocity [rotations per minute]

Figure 2: Contribution of the closest wind turbine to the sound pressure level measured at the second microphone closest to the house. Estimations and interval errors are made from forced stand still and plotted as a function of angular blade velocity.

\begin{tabular}{|c|c|c|}
\hline Wind direction & Fluctuation-indicator [dB(A)] & Background [dB(A)] \\
\hline N & 33.6 & 38.2 \\
NE & 31.4 & 40.0 \\
E & 25.2 & 40.9 \\
SE & 25.7 & 44.9 \\
S & 24.6 & 45.5 \\
SW & 26.3 & 42.7 \\
W & 31.3 & 40.6 \\
NW & 30.9 & 41.1 \\
\hline
\end{tabular}

Table 1: Median fluctuation-indicator and mean background noise level per wind direction.

model results in a quite satisfying adjusted $R^{2}$ of 0.73 , it is used to predict the background levels included later on in Section 4.4.1.

\subsubsection{Periodic noise part}

To find a measurable parameter for quantifying the periodic or fluctuating character of wind turbine noise, the Fourier spectrum of the $\frac{1}{3}$-octave band $\frac{1}{8}$-seconds time series is taken after removing the mean amplitude. The time interval for obtaining this spectrum is set to one minute, short enough to capture the periodic part in background noise. Furthermore, it has to be long enough to actually include the fluctuations. As explained previously in Section 4.1.1, the frequency of the periodic part of the amplitude is expected to be below $1 \mathrm{~Hz}$ given the operational maximum of 19.5 rotations per minute. With a one-minute time interval, the frequency resolution is $\frac{1}{60} \mathrm{~Hz}$ which was found to be sufficient. Additionally, many disturbing sounds such as a car or plane passage take less than one minute and can thus be removed by the spectral analysis. 


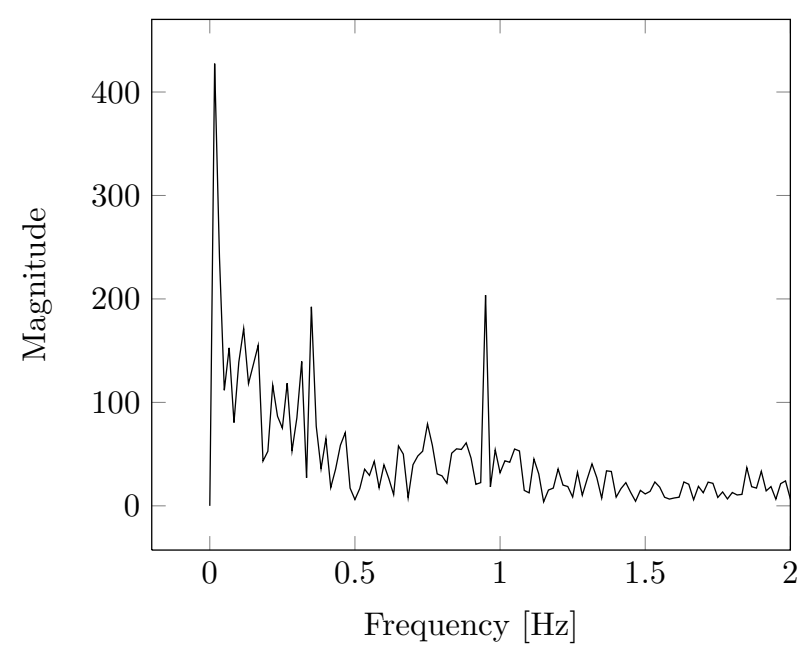

Figure 3: Spectrum of the amplitude of the A-weighted sound pressure level over time, calculated to quantify te fluctuating character of wind turbine noise (measurement taken on April 11th at 11:18 at the second measurement point closest to the house, 19 rotations per minute).

Figure 3 shows an example of the spectrum that is obtained. A clear peak just below $1 \mathrm{~Hz}$ is visible, indicating a frequency corresponding to a rotation speed of 19 rotations per minute. Often, there is a slight difference between the rotational speed obtained from the noise level fluctuation spectrum and the production data, which could be explained by the change in angular blade velocity during a single minute. In the case of the example, the production data rotation speed is 18.5 rotations per minute one minute before the measurement and 19 rotations per minute one minute after. To further summarize the many thousands of minutes of sound level data, the spectral level at the frequency corresponding to the production data angular blade velocity is selected, yielding to a so-called 'fluctuation-indicator'.

Field observations reveal that the fluctuating noise is more audible for higher angular blade velocity, but the overall statistical correlation between those two variables is very low because the periodic character is only present in certain distinct conditions, not only depending on the rotational speed.

By contrast, as explained previously in Section 2, the fluctuating character of wind turbine noise is closely related to directivity. When plotting the directivity patterns calculated from the work by Oerlemans [13] on a local map for the three wind turbines at the site under study, it can be seen that there are wind directions where the oscillation will coincide for the three turbines, which could lead to slow increase and decrease of the oscillating sound. This situation would theoretically occur mainly for northerly winds $\left(-26^{\circ}\right.$ to $\left.10^{\circ}\right)$ and to some extent for westerly winds $\left(244^{\circ}\right.$ to $\left.280^{\circ}\right)$ winds. For dwellings located in other direction, such situations would not occur. Specifically at the microphones' location, the highest fluctuation strength is theoretically expected for North-North-East $\left(-3^{\circ}\right.$ to $\left.33^{\circ}\right)$ wind and to a lesser extent for North-West-West $\left(267^{\circ}\right.$ to $\left.303^{\circ}\right)$ wind.

The median fluctuation-indicator per wind direction tabulated in Table 1 confirms to some extent the theoretical prediction. Again only observations with angular blade velocity of at least 10 rotations per minute are taken into account to avoid artifacts 
when the wind turbines rotate too slowly to actually cause noise. Additionally, a nonparametric Kruskal-Wallis test [22] (the assumption of normally distributed standardized residuals needed for ANOVA are not fulfilled) reveals that wind direction indeed has a statistical significant influence on the fluctuation-indicator $(p<0.001)$.

\subsubsection{Tonal component}

Studies of environmental noise exposure in general have shown that the presence of tonal components increases the risk of annoyance [25]. In wind turbines, tonality is rarely encountered and in-depth analysis of the sound recordings performed within this study do not provide any evidence for tonal components. This is in accordance with the fact that the researchers never observed tonality on site and with the manufacturers' statement that no tonal components have ever appeared for this type of wind turbines.

\subsection{Annoyance reports}

Three of the eight participating families report regularly, resulting in 552 reports in total. From the five non-respondents, three were willing to take the telephonic interview. Reasons for non-responding are the lack of annoyance-although the web-application accepted 'not annoyed'-reports-for one person, the other two families are from time to time annoyed by the turbines but had not found time to report. Finally, the Fisher's exact test for count data reveals no statistical difference in noise sensitivity between respondents and non-respondents $(p>0.05)$.

A global view of the response rate shows no clear changes over the four-months test time, the three respondents appear to fulfill the task consistently. In addition, no clear week-weekend or day-evening patterns are observed, possibly because there is only one household where both partners have daytime jobs.

By contrast, the three families apply clearly different response strategies; one household only reports when they are actually annoyed, one reports regularly-also when there is no annoyance-and the third follows an intermediate approach. These strategies are confirmed by the participants themselves during the debriefing.

\subsection{Operational and meteorological data and annoyance}

Annoyance is first investigated as a function of parameters that can be provided for every installations and hence could be a valid starting point for practical steering protocols. Logistic regression is carried out with the risk of high annoyance-i.e. highly and extremely annoyed-as outcome variable, analogous to general practice in environmental noise assessment [26]. Possible independent variables are chosen from the production and meteorological data (see Section 3.3). A manual step-forward procedure is followed, adding preferably parameters with minimal statistical correlation to the other independent variables.

This finally leads to a model with three independent parameters predicting the risk of high annoyance $(\mathrm{P}(\mathrm{HA}))$ : the angular blade velocity $\omega$, the categorized nacelle position i.e. the wind direction $Q$ (North, North-East, East, South-East, South, South-West, West, North-West) and the relative humidity $\rho$ (in \%), or as a formula

$$
\begin{gathered}
\mathrm{P}(\mathrm{HA})=\frac{1}{1+\exp (-X \hat{\beta})} \\
X \hat{\beta}=-6.89+0.601 \cdot \omega+\mathbf{Q} \cdot \boldsymbol{\delta}-0.0236 \cdot \rho
\end{gathered}
$$




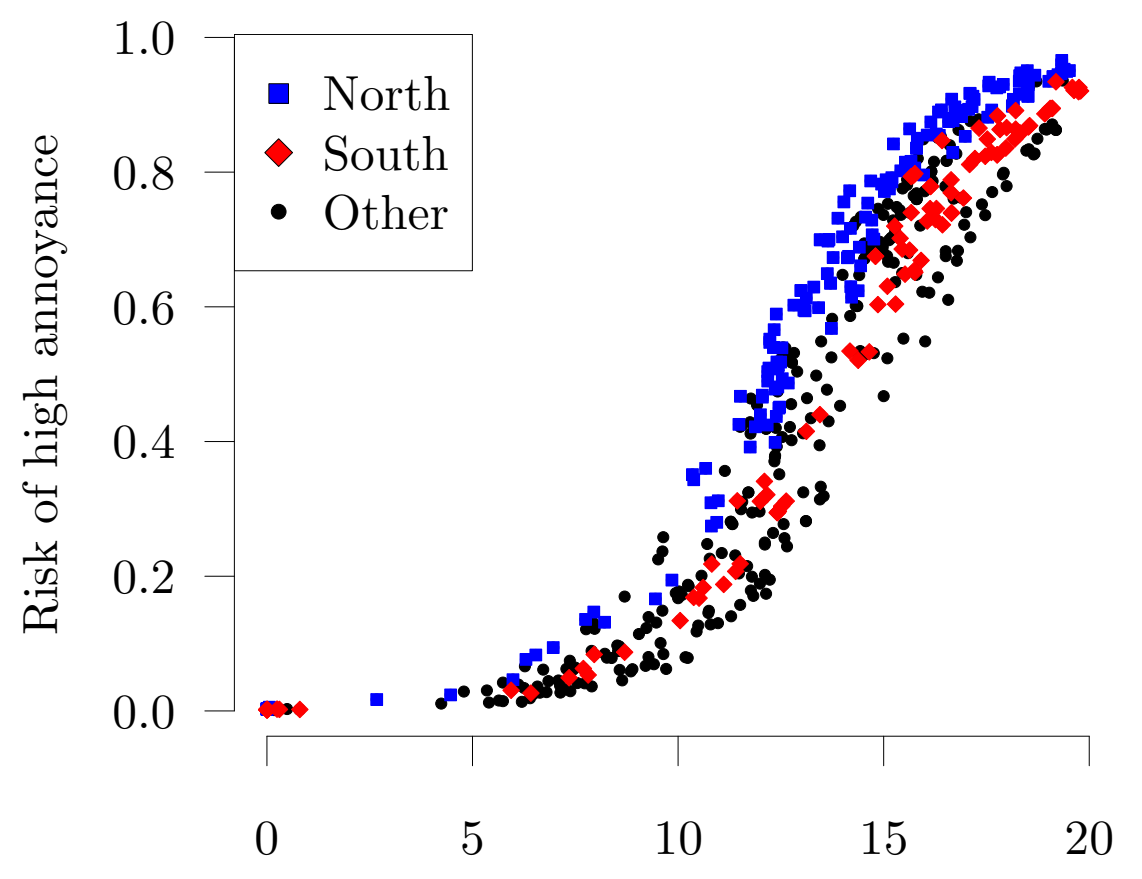

Angular blade velocity [rotations per minute]

Figure 4: Predicted risk of high annoyance as a function of angular blade velocity, taking into account the wind direction and relative humidity. Predictions for northern and southern wind are colored to emphasize the most extreme conditions for risk of high annoyance as a function of wind direction. Predictions for wind coming from NorthEast, East, South-East, South-West, West, North-West (labeled 'Other') are regardless of wind direction printed as black dots.

with

$$
\begin{gathered}
\mathbf{Q}=[-0.187,-0.334,+0.000,-0.791,-1.880,-1.384,-1.074,-1.025], \\
\boldsymbol{\delta}=[\{N\},\{N E\},\{E\},\{S E\},\{S\},\{S W\},\{W\},\{N W\}]^{T}
\end{gathered}
$$

and

$$
\{c\}=1 \text { if subject is in group } c, 0 \text { otherwise. }
$$

The risk of high annoyance especially increases with increasing angular blade velocity $(p<0.0001)$ followed by the wind direction $(p<0.001)$. Figure 4 shows that the probability for high annoyance is highest for wind coming from the North and lowest from southern wind. The results for wind coming from the East should not be taken into account due to lack of data.

Finally, the risk of high annoyance increases with decreasing relative humidity $(p<0.001)$. From the air absorption effect on sound, a higher sound pressure level is expected with increasing humidity [18], but this is not consistent with the observed decrease of annoyance. Hence, it seems most likely that the apparent effect is not related to sound propagation, but perhaps to the weather as such. 


\subsection{Annoyance, noise and steering parameters}

The previous analysis could provide operational restrictions for this particular site so that the risk of high annoyance can be reduced. However, they do not increase insight in underlying mechanism of noise annoyance, i.e. in noise characteristics related to annoyance. Hence, in this section the influential independent variables found above will be linked to possible important noise features.

\subsubsection{Noise and wind direction}

Section 4.1.3 and 4.1.4 have elaborated that background noise levels and the periodic part of the noise are related to the wind direction. Furthermore, these noise parameters might also influence the perceived annoyance; more pronounced fluctuations increase noticeability and possibly annoyance whereas higher background levels might have the opposite effect due to informational masking [8, 27].

It is verified with logistic regression whether these assumed effects can be formalized by replacing the variable wind direction in the previous model (Equation 2 ) by the predicted background levels $\widehat{L_{\mathrm{A} 95}}[\mathrm{~dB}(\mathrm{~A})]$ (Section 4.1 .3 and the median fluctuationindicator $\hat{\phi}[\mathrm{dB}(\mathrm{A})]$ (Section 4.1.4). This yields for the general Equation 1 to

$$
X \hat{\beta}=-7.893+0.647 \cdot \omega+0.0853 \cdot \hat{\phi}-0.0944 \cdot \widehat{L_{\mathrm{A} 95}}-0.0243 \cdot \rho
$$

with coefficients consistent with the expected effects. The angular blade velocity remains the strongest variable in this model $(p<0.0001)$, followed by the relative humidity $(p<0.01)$. For the newly added variables, the background level $(p<0.05)$ has a somewhat stronger influence then the fluctuation-indicator $(p<0.05)$, but in general both variables are strongly inversely correlated (Spearman $\rho=-0.45 ; p<0.0001$ ), making it difficult to disentangle their separate influence.

Compared to the previous model (Equation 2), both expressions are very similar in terms of AIC (434.8 for Equation 2 and 434.0 for Equation 3), and ROC curve, suggesting that combination of background level and fluctuation-indicator codes for almost all site specific annoyance-effects captured by the variable wind direction.

\subsubsection{Noise and angular blade velocity}

Specific noise levels emitted by the wind turbine are closely related to the angular blade velocity (see Section 4.1.2). To investigate their relationship with annoyance, specific emission levels $\widehat{L_{s}}[\mathrm{~dB}(\mathrm{~A})]$ are used in Equation 3 instead of the angular blade velocity. For the regression analysis, only observations with minimal 10 rotations per minute are retained because calculations are less accurate for lower angular blade velocity (again see Section 4.1.2, yielding to 428 data points. This then gives

$$
X \hat{\beta}=-13.1+0.253 \cdot \widehat{L_{s}}+0.157 \cdot \hat{\phi}-0.0269 \cdot \rho .
$$

Similar to the angular blade velocity in previous analyses, the specific emission level is now the strongest variable $(p<0.0001)$, followed by the fluctuation-indicator $(p<0.0001)$ and relative humidity $(p<0.0001)$. Background levels no longer have a statistical significant contribution, but once more (see Section 4.4.1) the strong correlation with the fluctuation-indicator might be an important issue. In terms of AIC, the latest model performs slightly better (422.5 versus 424.14 ) then the one with angular blade velocity (Equation 4 applied to the reduced dataset of 428 observations with minimal 10 rotations per minute) and both ROC curves almost overlap. 


\subsubsection{Annoyance and fluctuation-indicator}

Up to this point, the periodic character of wind turbine noise has been estimated from an average value per wind direction. However, unlike the background level and the specific emission level, this indicator can actually be measured continuously. Hence, exact values are available per annoyance report, possibly revealing an even more tight relationship between fluctuation and high annoyance than the estimated fluctuationindicator $\hat{\phi}$. Here, the maximum fluctuation-indicator $\phi$ in the 10-minute time interval of each annoyance report is used. Only observations in which the angular velocity is at least 10 rotations per minute are taken into account, because for lower blade velocity unrealistic outliers are present. Figure 5 illustrates that higher levels of annoyance correspond with an increase in the fluctuation-indicator.

Due to measurement equipment restrictions and missing data, the available data set is reduced to 301 observations by including the maximum fluctuation-indicator. To retain sufficient observations per independent variable, the candidate parameters are therefore limited to noise-related measures, leading to

$$
X \hat{\beta}=-7.27+0.155 \cdot \widehat{L_{s}}+0.00917 \cdot \phi .
$$

Unlike the background level $\widehat{L_{\mathrm{A} 95}}$, both $\widehat{L_{s}}$ and $\phi$ are statistical significant $(\alpha=$ 0.05 ), although the specific emission level remains the most important parameter for the fit. Nevertheless, compared to the estimated fluctuation-indicator $\hat{\phi}$, the maximal fluctuating-indicator $\phi$-being the actual fluctuation-strength at the time of the annoyance report - has a stronger influence on the statistical modeling and slightly improves the AIC (from 344.4 to 342.62 ).

\subsection{Balance between production and annoyance}

It goes without saying that quality of living should be guaranteed as much as possible when installing new wind turbines. But renewable energy sources might also contribute to a more sustainable way of living, hence wind turbines can not simply be banned, but instead a balance should be sought between lowering annoyance and preserving sufficient energy production.

This section will propose to optimize the operational restrictions based on wind direction and wind speed. The aim is to obtain the highest production possible for an allowed risk of high annoyance, or reciprocally the least risk of high annoyance guarantying a given production. The full 6-month dataset of all production and meteorological data is used to compare the impacts of the different limitation schemes on both production and risk of high annoyance.

Figure 6 compares the mean production as a function of the mean of risk of high annoyance. It should be read as follows: if the angular blade velocity is limited to 12 rotations per minute regardless of wind direction, an average production of almost $200 \mathrm{~kW}$ can be expected, and neighbors will risk high annoyance $8.1 \%$ of the time. The gray line represents the best limitation scheme corresponding to the dataset, which is the limitation per wind direction that provides the highest production, allowing a given mean risk of high annoyance. For example, replacing the limitation of 14 rotations per minute by a limitation of 11 for north wind and no limitation in other wind directions allows the mean risk of high annoyance to decrease from $16.7 \%$ to $13.7 \%$, while production is increased by $7.8 \%$. Further optimization reveals that production benefits of more than $10 \%$ can be obtained-retaining a certain risk of high annoyance-if 


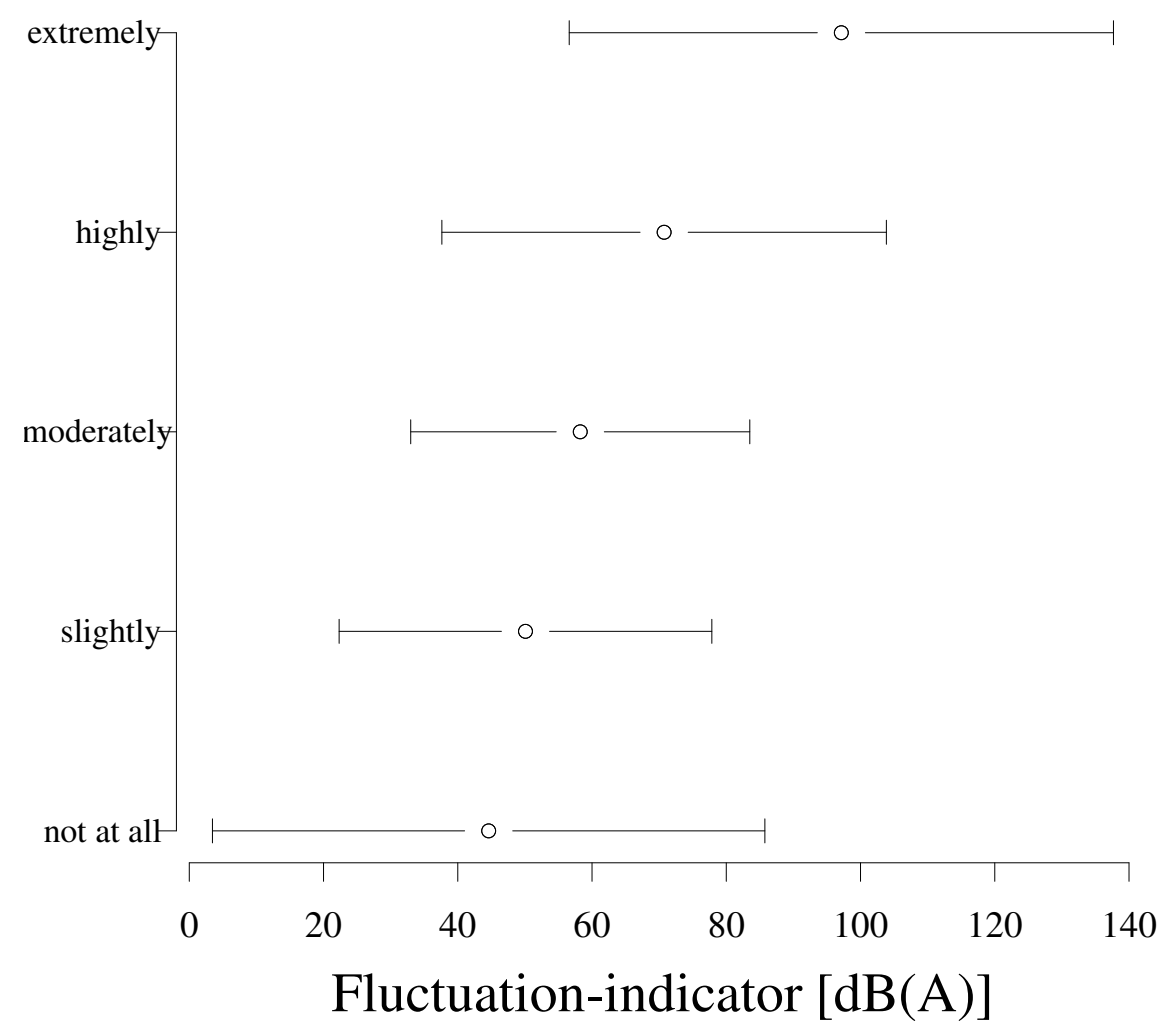

Figure 5: Average maximal fluctuation-indicator (plus or minus one standard deviation) per reported annoyance level. 


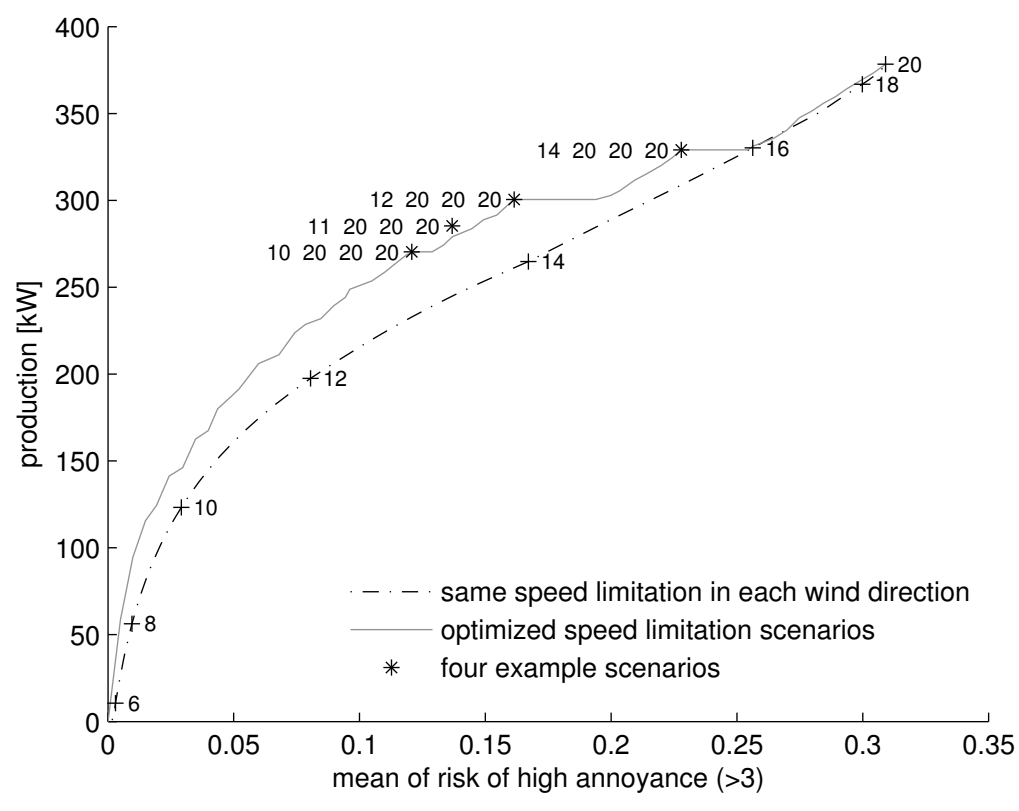

Figure 6: Production as a function of mean of risk of high annoyance; rotation per minute limitations are given for North, East, South and West, respectively.

operational limits between 11 and 14 rotations per minute are replaced by wind dependent limitations. Unsurprisingly, required limitations are stronger for north winds (see Section 4.3.

\section{Discussion}

In this paper, a multi-perspective approach is chosen to address the influence of wind turbines on the sonic environment. Combining sound measures with human auditory perception is more and more accepted as good practice for soundscape assessment and design [28, 29]. More specific, long-term recordings of numerous variables are combined with frequent annoyance assessment, making this almost an experimental setup-with extensive knowledge of varying input parameters-in a home environment.

The major limitation of the research carried out is the limited number of active participants. Although they have reported annoyance consequently during the test period, their representativeness for the larger community might be questionable. The main issue is whether the other neighbors are fundamentally not annoyed, or whether their choice not to participate is inspired by other factors.

First, detailed assessment of annoyance over time comes at a cost, being much more demanding than for instance one single interview. This might put some people off. Furthermore, the wind turbines are not undiscussed in this particular area. The public has no direct economical benefit from the turbines themselves, but a lot of neighbors and/or their relatives work in the factory the turbines are built for. Hence, considerations like employment might influence the attitude toward noise production. It has been shown that economical benefit does not affect the perception of wind turbine noise, but 
it largely decreases the reported annoyance [3]. Finally, the risk of annoyance might be objectively higher for certain inhabitants given the theoretical directivity of noise fluctuating actually found in this study. These considerations suggest that the reported noise annoyance should not be trivialized to hypersensitivity of the people effectively responding. Moreover, the correspondence with larger-scale studies supports the idea that the current findings are to a certain extent transferable [3].

The paper at hand aims to relate the risk of high annoyance with operational, meteorological and noise data. In this regard, [17] has shown that the propagation parameters wind speed and wind direction largely influence respectively the source strength and the sound immission. This supports the finding in Section 4.3 that wind direction and angular blade velocity are statistically significant related to the risk of high annoyance. Here, angular blade velocity is a better measure than wind speed due to the close relationship with specific turbine noise emission.

Other propagation parameters like temperature at hub height and temperature gradient [17] could not be adopted to risk of annoyance, while humidity-not included as an important parameter in [17]-appears statistically significant but inversely to the effect expected on propagation grounds. Different weather conditions between [17] and the current study might play a small role, but for annoyance the effect of weather on people's behavior is probably much more important than on sound propagation since temperature and humidity account for at most $1 \mathrm{~dB}(\mathrm{~A})$ in sound level difference according to [18]. Here, one could expect that people are more exposed when dryer weather allows them to open windows and spend time outside, possibly increasing the risk of annoyance. Temperature would probably be more important if the test period includes summertime and not only spring.

This study also aims to establish alternative noise parameters to address annoyance. Wind turbine specific emission and fluctuation clearly increase the risk of high annoyance, whereas higher background noise slightly lowers it. The close relationship between the fluctuation-indicator and noise annoyance found in this study suggests that current quantification corresponds to a certain extent to the qualitative descriptions reported earlier [10]. Background noise appears somewhat less strong in the statistical analysis and also in literature there exists some ambiguity; [30] could not establish masking effects of wind noise on subjective rating of wind turbine noise, whereas [8] states that natural background noise has positive effects on perceived loudness, although the in-field effects are yet under study [31]. Here, the established regression model is unable to pronounce upon possible causal relationships between annoyance, background level and fluctuation and even the strength of the parameters' individual influence has to be interpreted with caution since the two noise measures are correlated and possibly coding for underlying factors.

Finally, linking annoyance to quantitative parameters might be useful to steer the wind turbines so that annoyance can be reduced. Although there is some skepticism about the real effects of operational restrictions [30], nighttime bans are already considered good practice [3]. All this suggests that an extension of the restrictions period will decrease the annoyance further. In accordance to [32], the current research shows that operational restrictions and cost-effectiveness are not necessary unconciliatory if steering is done in a more sophisticated way by taking variables like wind direction into account. Nevertheless, one should bear in mind that this remains a post-hoc measure, by no means replacing technical improvement or efforts necessary to form a positive attitude before the wind turbines are erected [33].

For further research, the on-line report tool could be applied on a larger scale to address transferability of the independent variables found in this study, together 
with the benefits from operational restrictions depending on wind direction and/or the fluctuation-indicator's strength. In addition, the nature of the relationship between annoyance, fluctuation-indicator and background noise could be addressed in more controlled laboratory conditions.

\section{Conclusion}

The current study confirms once more that annoyance due to wind turbine noise is a complex matter where various factors have to be taken into account. Not only personal and contextual variables, but also noise production and propagation itself might cause substantial differences in annoyance reported in a particular region.

Detailed noise recordings and spectral registrations allow to calculate the additional emission caused by wind turbine noise and the background noise level, in addition a so-called 'fluctuation-indicator' is established to account for the periodic character of wind turbine noise. The wind turbine specific emission can clearly be linked to its operational characteristics whereas the fluctuation-indicator is more closely related to directivity and wind direction.

Directivity also plays its role in noise annoyance since risk of high annoyance is determined by angular blade velocity together with wind direction. Hence, more subtle steering protocols - not only based on a certain number of rotations per minute-might reduce noise annoyance while preserving cost-effectiveness.

Furthermore, studying noise exposure directly is necessary to gain insight in underlying noise annoyance mechanisms. Specific emission plays a very important role, in addition the periodic character of the noise (captured by the fluctuation-indicator) can not be neglected. Finally, background levels appear influential to a certain extent, but the relative importance of the different noise parameters should be interpreted with caution due to their mutual correlation.

\section{Acknowledgement}

Bert De Coensel and Annelies Bockstael are postdoctoral fellows of the Research Foundation-Flanders; the support of this organization is gratefully acknowledged.

\section{References}

[1] Directive 2009/28/EC of the European Parliament and of the Council of 23 April 2009 on the promotion of the use of energy from renewable sources and amending and subsequently repealing Directives 2001/77/EC and 2003/30/EC. European Parliament and Council, Brussels (2009).

[2] A. Van Rompaey, S. Schmitz, C. Kesteloot, K. Peeters, B. Moens, et al.: Landscape capacity and social attitudes towards wind energy projects in Belgium (2010).

[3] E. Pedersen, F. van den Berg, R. Bakker, J. Bouma: Response to noise from modern wind farms in The Netherlands. The Journal of the Acoustical Society of America 126 (2009) 634. 
[4] A. Salt, T. Hullar: Responses of the ear to low frequency sounds, infrasound and wind turbines. Hearing research (2010).

[5] E. Pedersen, P. Larsman: The impact of visual factors on noise annoyance among people living in the vicinity of wind turbines. Journal of Environmental Psychology 28 (2008) 379-389.

[6] E. Pedersen, F. van den Berg, R. Bakker, J. Bouma: Can road traffic mask sound from wind turbines? Response to wind turbine sound at different levels of road traffic sound. Energy Policy 38 (2010) 2520-2527.

[7] S. Janssen, H. Vos, A. Eisses, E. Pedersen: A comparison between exposureresponse relationships for wind turbine annoyance and annoyance due to other noise sources. Journal of the Acoustical Society of America 130 (2011) 3746.

[8] K. Bolin, M. Nilsson, S. Khan: The potential of natural sounds to mask wind turbine noise. Acta Acustica united with Acustica 96 (2010) 131-137.

[9] K. Persson, E. Öhrström: Psycho-acoustic characters of relevance for annoyance of wind turbine noise. Journal of sound and vibration 250 (2002) 65-73.

[10] E. Pedersen, K. Waye: Wind turbines-low level noise sources interfering with restoration? Environmental Research Letters 3 (2008) 015002.

[11] E. Pedersen, H. Halmstad: Noise annoyance from wind turbines: A review (2006).

[12] S. Wagner, R. Bareib, G. Guidati: Wind Turbine Noise. Springer, Berlin (1996).

[13] S. Oerlemans: Detection of aeroacoustic sound sources on aircraft and wind turbines. Ph.D. thesis, University of Twente (2009).

[14] S. Oerlemans, P. Sijtsma, B. Méndez López: Location and quantification of noise sources on a wind turbine. Journal of sound and vibration 299 (2007) 869-883.

[15] G. Van den Berg: Wind turbines at night: acoustical practice and sound research. In Proceedings Euronoise 2003.

[16] G. Van den Berg: Effects of the wind profile at night on wind turbine sound. Journal of Sound and Vibration 277 (2004) 955-970.

[17] J. Forssen, M. Schiff, E. Pedersen, K. Waye: Wind turbine noise propagation over flat ground: Measurements and predictions. Acta Acustica united with Acustica 96 (2010) 753-760.

[18] ISO 9613-2:1996 Acoustics - Attenuation of sound during propagation outdoors - Part 2: General method of calculation. Internation Standards Organisation (1996).

[19] ISO/TS 15666:2003 Acoustics - Assessment of noise annoyance by means of social and socio-acoustic surveys. Internation Standards Organisation (2003).

[20] N. Weinstein: Individual differences in reactions to noise: A longitudinal study in a college dormitory. Journal of Applied Psychology 63 (1978) 458-466. 
[21] E. Pedersen, K. Waye: Perception and annoyance due to wind turbine noise-a dose-response relationship. The Journal of the Acoustical Society of America 116 (2004) 3460.

[22] M. H. Kutner, C. J. Nachtscheim, J. Neter, W. Li: Applied linear statistical models. McGraw-Hill, New York, 5th edition (2004). 1396 pages.

[23] D. Bates, M. Maechler, B. Boker: Linear mixed-effects models using S4 classes: Package 'lme4' (2011).

[24] D. Bailey, F. Alimadhi: logit.mixed: Mixed effects logistic model. In K. Imai, G. King, O. Lau, eds., Zelig: Everyone's Statistical Software (2007).

[25] J. Kang: Urban sound environment. Taylor \& Francis, Oxon (2007).

[26] H. Miedema, H. Vos: Exposure-response relationships for transportation noise. The Journal of the Acoustical Society of America 104 (1998) 3432.

[27] B. De Coensel, D. Botteldooren, T. De Muer, B. Berglund, M. Nilsson, et al.: A model for the perception of environmental sound based on notice-events. The Journal of the Acoustical Society of America 126 (2009) 656-665.

[28] R. M. Schafer: The soundscape: Our Sonic Environment and the Tuning of the World. Destiny Books, Rochester, Vermont, USA (1994).

[29] B. De Coensel, D. Botteldooren: The quiet rural soundscape, and how to characterize it. Acta Acustica united with Acustica 92 (2006) 887-897.

[30] B. Hoen: Assessing the Impacts of Reduced Noise Operations of Wind Turbines on Neighbor Annoyance: A Preliminary Analysis in Vinalhaven, Maine (2010).

[31] E. Pedersen, F. van den Berg: Why is wind turbine noise poorly masked by road traffic noise? In Proceedings Internoise 2010. SPA, SEA, Lisboa (2010).

[32] F. Cotana, F. Rossi, M. Filipponi: Economics on wind farm noise mitigation by power limitation. In Proceedings of 20th International Congress on Acoustics, ICA 2010. Sydney, Australia (2010).

[33] M. Wolsink: Planning of renewables schemes: Deliberative and fair decisionmaking on landscape issues instead of reproachful accusations of noncooperation. Energy Policy 35 (2007) 2692-2704. 